\title{
Algunas observaciones sobre la sinterización del acero austenítico 316L en atmósfera de argón
}

\author{
F. Gómez ${ }^{(*)}$, J.M. Gallardo $^{(*)}$ y E.J. Herrera ${ }^{(*)}$ \\ Resumen La utilización de acero inoxidable pulvimetalúrgico está limitada, entre otras razones, por la presen- \\ cia de porosidad que pueda favorecer procesos corrosivos, así como a posibles pérdidas de cromo en \\ la matriz durante los procesos de sinterización. Se han realizado experiencias de procesado de polvos \\ de acero 316L con distintas presiones de compactación (100 - 1.000 MPa), temperaturas de sinteri- \\ zación $\left(1.100-1.250^{\circ} \mathrm{C}\right)$, tiempos de sinterización $(15-240 \mathrm{~min})$ y en distintas atmósferas de sinte- \\ rización, de argón y vacío. Se obtiene una mejor densificación con elevada temperatura y tiempos de \\ sinterización en atmósferas de argón a baja presión. En dicha atmósfera, no se produce oxidación \\ superficial aislando parcialmente las muestras del flujo directo del argón.
}

Palabras clave: Pulvimetalurgia. Acero inoxidable. Oxidación superficial. Densificación.

\section{Some observations on the sintering of 316L austenitic steel in argon atmosphere}

\begin{abstract}
PM high speed steels are prone to higher corrosion rates due to residual porosity as well as chromium depletion of the matrix during sintering AISI $316 \mathrm{~L}$ powders have been cold compacted $(100-1,000$ $\mathrm{MPa})$ and sintered $\left(1,000-1,250^{\circ} \mathrm{C}, 15-240 \mathrm{~min}\right)$ under several argon containing or vacuum atmospheres. Better densification rates can be achieved as sintering time or temperature increase in the presence of argon. No surface oxidation has been observed with the use of low pressure argon atmospheres, as long as samples are protected in a stainless steel partially sealed container.
\end{abstract}

Keywords: Powder metallurgy. Stainless steel. Surface oxidation. Densification.

\section{INTRODUCCIÓN}

El acero AISI 316L constituye una de las opciones con mayor atractivo para la fabricación de piezas pulvimetalúrgicas inoxidables, entre otras razones, por su buena resistencia a la corrosión. Debe asegurarse una buena densificación y ausencia de oxidación durante el procesado. Las condiciones que aparecen aconsejadas en la literatura (1-7) comprenden ciclos de sinterización a temperaturas de $1.050-1.315^{\circ} \mathrm{C}$ en atmósferas de amoníaco disociado, vacío, argón a baja presión, etc. Las atmósferas de hidrógeno o de argón a baja presión producen una densificación óptima sin afectar a la resistencia a la corrosión del acero (5).

(*) Grupo de Metalurgia e Ingeniería de los Materiales. E.S.I. Avda. de los Descubrimientos s/n. 41092-Sevilla (España).
En este trabajo, se pretende preparar muestras que, posteriormente, puedan ser sometidas a ensayos de corrosión, tal como resultan de la sinterización. Para ello, ha sido necesario determinar las condiciones de procesado que permitan obtener una sinterización suficiente, así como superficies limpias de óxidos a la salida del horno.

\section{PROCEDIMIENTO EXPERIMENTAL}

Se prepararon muestras cilíndricas de $12 \mathrm{~mm}$ de diámetro, así como probetas de tracción de geometría similar a la recomendada por MPIF (8). En la compactación mecánica en frío, se utiliza lubricación en pared con cera Hoechst Wachs C Micropulver. Para obtener distintos grados de porosidad final, se seleccionaron presiones de compactación 
de $100,400,700$ y $1.000 \mathrm{MPa}$. Las probetas, una vez compactadas, se deslubrican al aire a $425^{\circ} \mathrm{C}$ durante $1 \mathrm{~h}$. La sinterización se llevó a cabo con tiempos de 15, 60 y 240 min. Dichos tiempos son reales de permanencia a la temperatura seleccionada, ya que las probetas se introducen y extraen con el horno previamente calentado. Las temperaturas de sinterización empleadas fueron 1.100, 1.150, 1.200 y $1.250{ }^{\circ} \mathrm{C}$. El enfriamiento de las muestras se realizó en flujo de argón para evitar la precipitación de carburos en las mismas.

En cuanto a la atmósfera de sinterización, se seleccionó una de vacío con una pequeña presión de argón, lo que confiere a las piezas sinterizadas propiedades semejantes a las que se obtienen sinterizando en hidrógeno sin los problemas de seguridad que supone el uso de este gas. El valor elegido para la presión parcial de argón debe ser suficiente para evitar, en la medida de lo posible, la evaporación del cromo contenido en el acero inoxidable. Con los 0,8 mbar elegidos, puede evitarse parcialmente la evaporación del cromo, pero no la del óxido de cromo $\left(\mathrm{Cr}_{2} \mathrm{O}_{3}\right)$, para lo que se requerirían presiones altísimas a la temperatura de sinterización (9). Con objeto de disminuir la oxidación superficial de las muestras, se utilizaron diversos montajes experimentales, a saber: a) colocación de las muestras en flujo de argón calidad N45 (3 ppm de $\mathrm{H}_{2} \mathrm{O}$, 3 ppm de $\mathrm{O}_{2}$ ) (Fig. 1). b) aportación del argón entre el horno y el equipo de vacío, quedando las muestras en un tubo sin salida por el extremo opuesto. c) como en el primer caso, pero usando argón de calidad N60 (0,6 ppm de $\mathrm{H}_{2} \mathrm{O}, 0,1 \mathrm{ppm}$ de $\left.\mathrm{O}_{2}\right)$. d) como en el primer caso, usando además getters para el oxígeno $\left(\mathrm{Cr}\right.$ a $950{ }^{\circ} \mathrm{C}, \mathrm{Al}$ a $585^{\circ} \mathrm{C}$, Ti a $850^{\circ} \mathrm{C}$ y $\mathrm{Cu}$ a $600{ }^{\circ} \mathrm{C}$ ) y el agua (perclorato de magnesio), colocados en la corriente de argón antes de pasar sobre las probetas y e) como en el primer caso, pero protegiendo las probetas dentro de un contenedor de acero inoxidable semiestanco.

Además, se probó una atmósfera estacionaria de 1,25 bar de argón y vacío (2,1 E-2 mbar). En las condiciones descritas en los párrafos anteriores, se

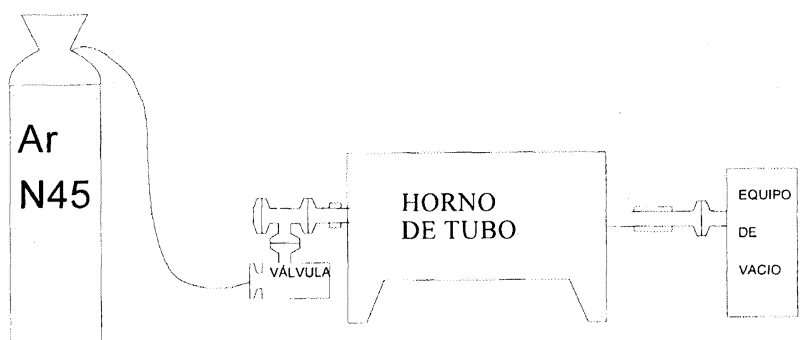

FIG. 1.- Disposición de los equipos en la primera prueba de sinterización.

FIG. 1.- Sintering equipments arrangements as used in the first sintering test. prepararon probetas en las que se determinaron las propiedades finales de densidad, dureza, carga de rotura, alargamiento y oxidación superficial.

\section{RESULTADOS Y DISCUSIÓN}

\subsection{Densidad final}

En general, puede destacarse que la densidad final aumenta al usar presiones, temperaturas y/o tiempos de sinterización mayores, dentro de los márgenes de presiones, temperaturas y tiempos utilizados.

La densidad de las probetas sinterizadas depende principalmente de la presión de compactación, en menor medida del tiempo y, por último, de la temperatura de sinterización. También la atmósfera de sinterización afecta a la densificación obtenida. Se alcanzan los mejores resultados con atmósfera dinámica -montaje (a)- de 0,8 mbar de argón. En general, siempre que exista argón en la atmósfera, se produce una buena densificación, por lo que lá sinterización con una atmósfera de 1,25 bar de argón produce resultados sólo algo peores. Al sinterižár en vacío (2,1 E-2 mbar), se produce una densificación inferior a los casos anteriores. No aparecen diferencias significativas al variar otros parámetros, como el procedimiento de introducción de las muestras, calidad del argón, etc. Los valores de la densidad final obtenida varían entre 5,89 y 7,31 $\mathrm{g} / \mathrm{cm}^{3}$ (74,55 y $92,53 \%$, respectivamente).

\subsection{Propiedades mecánicas}

En general, la dureza $\mathrm{HV}_{10}$, carga de rotura y alargamiento aumentan al usar presiones, temperaturas y/o tiempos de sinterización mayores, dentro de los márgenes de presiones, temperaturas y tiempos utilizados.

La dureza depende principalmente de la presión de compactación, con variaciones entre 16 y 103 $\mathrm{kg} / \mathrm{mm}^{2}$.

En la carga de rotura, la influencia de las tres variables de sinterización es igualmente importante, obteniéndose valores comprendidos entre 137 y 436 $\mathrm{MPa}$.

Para el alargamiento, se hace muy importante la influencia de la temperatura y el tiempo de sinterización, con valores que oscilan entre 0,85 y $26,85 \%$.

\subsection{Oxidación superficial}

En cuanto a la oxidación superficial de las muestras, tal como se extraen del horno de sinterización, la influencia más importante es la de la 
atmósfera y la del montaje del equipo de gases/ vacío utilizado.

Los montajes (a), (b) y (c) dan lugar, en mayor o menor grado, a una oxidación apreciable a simple vista. Sólo el uso de getters -montaje (d)- conduce a leves mejoras, aunque las probetas siguen mostrando, microscópicamente, la presencia de óxidos superficiales (Fig. 2a). Únicamente en la zona de las muestras en contacto con la navecilla cerámica en la que se colocaban para introducirlas en el horno, se observó que en muchas ocasiones la oxidación superficial era menor. En el montaje (e), que evita que el argón incida directamente sobre las muestras, desde el punto de vista microestructural, no se observan óxidos (Fig. 2b).

Para comprobar los resultados observados en el microscopio, se ha realizado un estudio, en el microscopio electrónico de barrido, mediante EDX, en el que se analiza la composición final de las muestras (Cr, Ni y Mo) en función de la profundidad, a partir de donde termina la capa de óxido formada.

En las figuras $3 \mathrm{a}$ y $3 \mathrm{~b}$ se muestran los resultados de los análisis realizados en una probeta preparada con el montaje (d), con $\mathrm{Cr}$ a $950{ }^{\circ} \mathrm{C}$ como getter, y según el montaje (e), respectivamente. En el segundo caso, el análisis se realiza desde la misma superficie, ya que no se observa óxido en la misma.

De forma similar a lo representado en la figura 3a, en todas las pruebas de sinterización en las que aparece óxido superficialmente, se produce una sustancial pérdida de cromo en la matriz. Puede decirse que aproximadamente las primeras $50-100 \mu \mathrm{m}$ no son inoxidables $(\mathrm{Cr}<13 \%)$ y que sólo a una profundidad de 100 - $200 \mu \mathrm{m}$ se alcanza la composición típica del acero. Por su parte, el análisis EDX correspondiente a la figura $3 \mathrm{~b}$ indica que el montaje (e) evita cualquier pérdida de cromo desde la misma superficie de la muestra.

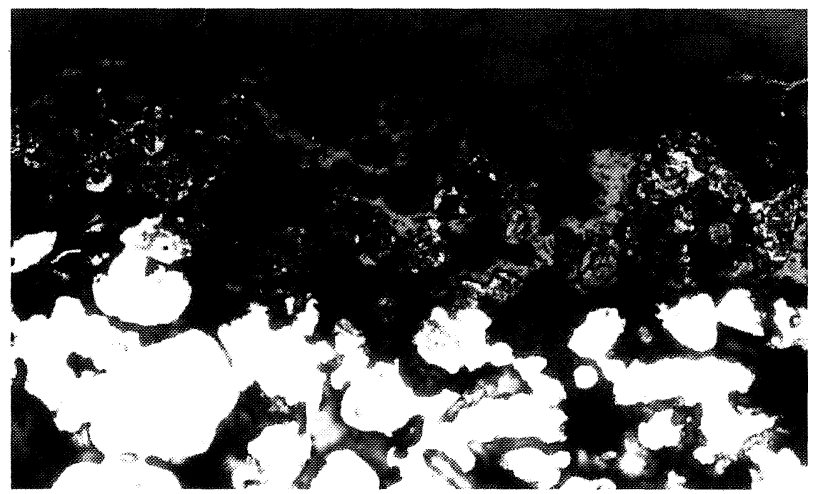

De los resultados anteriores se deduce que la oxidación solamente se elimina si se evita que el flujo de argón incida directamente sobre las muestras, no habiéndose observado ningún efecto negativo sobre la buena densificación típica de otros montajes con atmósfera de argón.

Para explicar los resultados anteriores, puede suponerse que la fuente de oxígeno que produce la oxidación del acero es el contenido en el propio argón, aunque no puedan descartarse las contaminaciones a través de los equipos o sus conexiones.

Cuando las probetas de acero inoxidable se someten a alta temperatura, el óxido de cromo formado tiene más tendencia a evaporarse que el cromo puro, por lo que la reacción global a considerar será:

$$
2 \mathrm{Cr}(s)+3 / 2 \mathrm{O}_{2} \leftrightarrow \mathrm{Cr}_{2} \mathrm{O}_{3}(\mathrm{~g})
$$

La constante de equilibrio de la reacción anterior es:

$$
K=\frac{P_{\mathrm{Cr}_{2} \mathrm{O}_{3}(g)}}{[\mathrm{Cr}]^{2} \cdot P_{\mathrm{O}_{2}}^{3 / 2}}
$$

siendo $P_{\mathrm{Cr}_{2} \mathrm{O}_{3}}$ y $P_{\mathrm{O}_{2}}$ las presiones parciales del óxido de cromo y del oxígeno, respectivamente.

En función de esta constante pueden explicarse los dos comportamientos distintos que se observan respecto al grado de oxidación: al recibir las probetas el flujo de argón de la atmósfera dinámica (montaje a) y al protegerlas mediante un tubo de acero inoxidable (montaje e).

Cuando las probetas se encuentran expuestas al flujo de argón, el óxido de cromo que se va evaporando en la superficie es arrastrado, por lo que su presión parcial disminuye, y la reacción tiende a

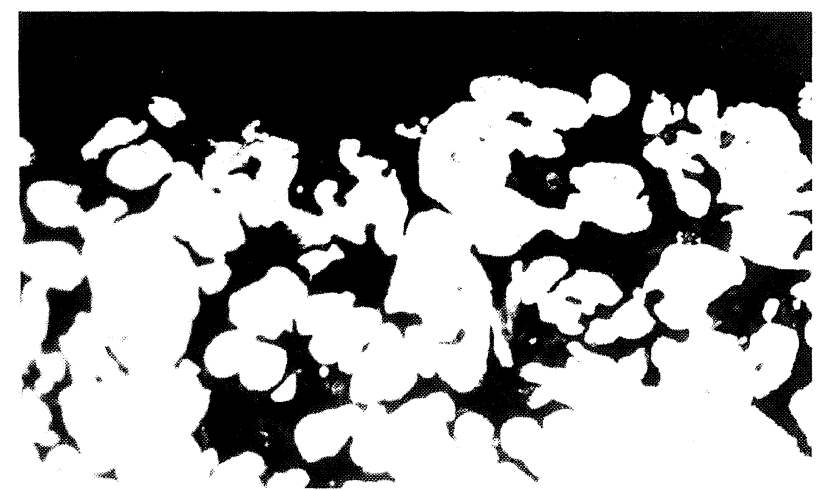

Fig. 2.- Microestructura superficial en probetas de $100 \mathrm{MPa}$ sinterizadas a $1.250{ }^{\circ} \mathrm{C}$ durante $1 \mathrm{~h}$, según el montaje (a) y (e), respectivamente $(\times 400)$.

FIG. 2.- Surface microstructure of samples compacted at $100 \mathrm{MPa}$ and sintered at $1,250{ }^{\circ} \mathrm{C}$ during $1 \mathrm{~h}$ with equipments arrangements $(a)$ and $(e)(\times 400)$. 

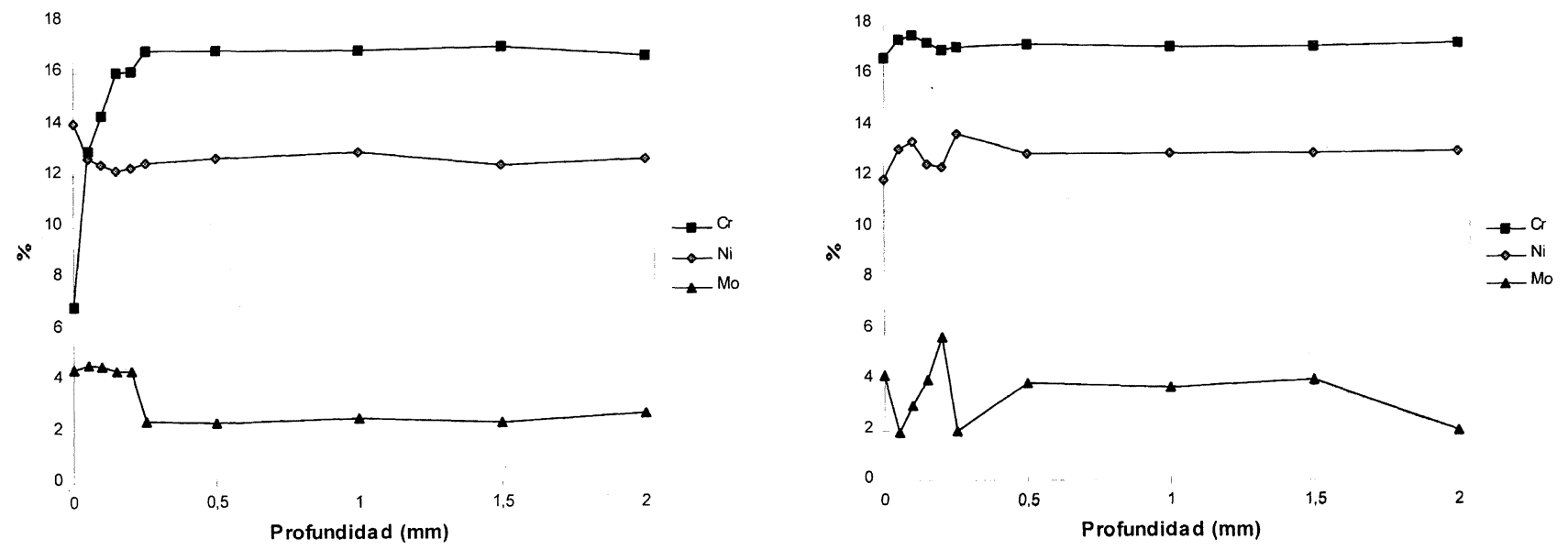

FIG. 3.- Análisis mediante EDX de probetas de $100 \mathrm{MPa}$ sinterizadas a $1.250{ }^{\circ} \mathrm{C}$ durante $1 \mathrm{~h}$ según el montaje (d) y (e), respectivamente.

FIG. 3.- EDX analysis of samples pressed at $100 \mathrm{MPa}$ sintered at $1,250{ }^{\circ} \mathrm{C}$ during $1 \mathrm{~h}$ with the equipment arrangements $(d)$ and $(e)$, respectively.

desplazarse a la derecha para tratar de restablecer el equilibrio.

La zona superficial tiende a oxidarse más que la interna, ya que en esta última la presión parcial del $\mathrm{Cr}_{2} \mathrm{O}_{3}$ no varía, al no ser arrastrado este compuesto tras evaporarse.

Otro factor que favorece la oxidación, cuando las probetas reciben el flujo de argón, es el aporte continuo de oxígeno a través del gas. El oxígeno se va renovando continuamente y la reacción de nuevo tiende a desplazarse a la derecha.

En el caso de estar las probetas protegidas con el tubo de acero inoxidable, el flujo de argón es externo a éste, por lo que no arrastra el $\mathrm{Cr}_{2} \mathrm{O}_{3}$ evaporado, incluso la presión parcial de éste puede aumentar y hacer que la reacción se desplace hacia la izquierda para restablecer el equilibrio.

Además, el aporte de oxígeno, en el caso de que las probetas se encuentren protegidas, es mucho menor, por lo que se evita una oxidación excesiva y, tras consumirse el oxígeno, la reacción tiende a desplazarse de nuevo a la izquierda.

Según se observa en la expresión de la constante de equilibrio, considerando los exponentes a los que se encuentran elevadas las presiones parciales, para evitar la oxidación, resulta más importante el efecto de la disminución de la presión parcial del oxígeno que el efecto de aumentar la presión parcial del óxido de cromo. Ahora bien, puede que ambos factores tengan influencia en los resultados obtenidos.

\section{CONCLUSIONES}

Se han realizado pruebas de sinterización en un acero inoxidable AISI 316L con el fin de obtener muestras válidas para un análisis de corrosión en el estado en que las mismas salen del horno. Para ello se han probado distintas presiones de compactación, temperaturas, tiempos y atmósferas de sinterización.

Se obtiene una densificación adecuada de las probetas sinterizando en atmósfera de argón a poca presión. La ausencia de oxidación superficial de las muestras se obtiene únicamente cuando las mismas se aíslan del flujo directo del argón, sin repercutir esto negativamente en la densificación.

\section{REFERENCIAS}

(1) Reinshagen, J.H. y Neupaver, A.J. Proc. Conf. on Advances in PM. Vol 2. San Diego, (CA, EE.UU.) Jun. 1989: 283-295.

(2) Stevenson, R.W. P/M Stainless Steel, Metals Handbook,

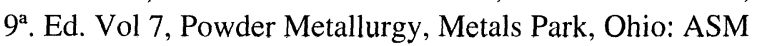
1984: $728-732$.

(3) Introduction to Powder Metallurgy. Catálogo de la European Powder Metallurgy Assoc., 1995: 13.

(4) Nayar, H.S. y Wasiczco, B. Metals Powder Report, Sep. 1990: $611-614$.

(5) Moyer, K.H. y Jones, W.R. Proc. 1991 AMPI/MPIF Powder Metallurgy Conf., Chicago (ILL., EE.UU.) 9-12 Jun. 1991: $145-148$.

(6) Frisk, K., Johansson, A. y LindberG, C. Proc. "PM 92 World Cong.", San Francisco (CA. EE.UU.) 21-26 Jun. 1992: 167-181.

(7) Beiss, P. Powder Metall., 34 (4), 1991: 259 - 261.

(8) Gallardo, J.M., Rodriguez, J.A. y Herrera, E.J. Anales de Mecánica de la Fractura. XII encuentro del Grupo Español de Fractura, La Coruña (España). Mar. 1995: 174 -179 .

(9) Kubaschewski, O., Evans, E.Ll. y Albock, C.B. Metallurgical Thermochemistry, $4^{\mathrm{a}} \cdot$ Ed: 409. 